Revue de droit comparé du travail et de la sécurité sociale

$3 \mid 2019$

Les migrations internationales de travail

\title{
Les cybermigrants, un concept juridique 4.0
}

\section{Amalia de la Riva}

\section{OpenEdition}

Journals

Édition électronique

URL : https://journals.openedition.org/rdctss/1466

DOI : 10.4000/rdctss. 1466

ISSN : 2262-9815

Éditeur

Centre de droit comparé du travail et de la sécurité sociale

Édition imprimée

Date de publication : 1 novembre 2019

Pagination : $72-79$

ISSN : 2117-4350

\section{Référence électronique}

Amalia de la Riva, « Les cybermigrants, un concept juridique 4.0 », Revue de droit comparé du travail et de la sécurité sociale [En ligne], 3 | 2019, mis en ligne le 01 novembre 2021, consulté le 13 novembre 2021. URL : http://journals.openedition.org/rdctss/1466 ; DOI : https://doi.org/10.4000/rdctss. 1466

\section{(c) (i) (9)}

Revue de droit comparé du travail et de la sécurité sociale est mise à disposition selon les termes de la Licence Creative Commons Attribution - Pas d'Utilisation Commerciale - Pas de Modification 4.0 International. 


\section{LES CYBERMIGRANTS, UN CONCEPT JURIDIQUE 4.0}

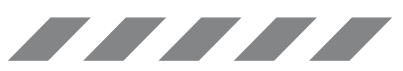

\section{ABSTRACT}

The Industrial Revolution 4.0 places us before a resizing of the way in which companies create value beyond borders. To the delocalisation of the production of material goods as a frequent behavior in the business strategy, now, it is possible to add a growing internationalization in the creation of value in the service sector of the economy. This new irruption, made possible by the development, improvement and use of information technologies, typical of the Industrial Revolution 4.0, confronts us with a new type of workers: cybermigrants. The present work proposes to expose a novel legal concept that allows to identify those workers who provide services from outside the company, abroad, providing direct response to the final clients demands. In short, providing instant consumption services, at the customer's seat, but from outside the borders. It also proposes a strategy to cover these subjects through the application of the international statute that has been built for migrant workers.

\section{KEY WORDS: Cybermigrants, Migrants, Industrial Revolution 4.0, Information Technologies, Transnational Services.}

\section{RÉSUMÉ}

La révolution industrielle 4.0 redéfinit la façon dont les entreprises créent de la valeur par-delà les frontières. À la délocalisation de la production de biens matériels, considérée comme une stratégie commerciale courante des entreprises, s'ajoute aujourd'hui l'internationalisation croissante de la création de valeur dans le secteur des services. Cette nouvelle percée, rendue possible par le développement, l'amélioration et l'utilisation des technologies de l'information caractéristiques de la révolution industrielle 4.0, a engendré un nouveau type de travailleurs: les «cybermigrants». Cet article expose un nouveau concept juridique pour identifier les travailleurs qui, par-delà les frontières de leur pays et en dehors de l'entreprise, fournissent des services en temps réel à des clients. La présente étude propose également une stratégie permettant de protéger ces travailleurs en leur appliquant le statut international de travailleurs migrants.

MOTS CLÉS: Cybermigrants, migrants, révolution industrielle 4.0, technologies de I'information, services transnationaux. 
a dénommée quatrième révolution industrielle s'inscrit dans le prolongement direct de la machine à vapeur, de l'électricité et de l'ordinateur. Elle repose sur la diffusion des technologies de l'information, ces dernières étant caractérisées par l'interconnexion massive des systèmes et des dispositifs numériques, mais aussi et surtout par l'intelligence artificielle qui combine une immense quantité de données (big data) et l'utilisation d'algorithmes pour leur traitement.

Chacune des révolutions industrielles précédentes a eu de fortes répercussions sur les modalités de production, et par-delà sur l'établissement des relations de travail et la gouvernance du système. L'impact fut tel que l'émergence du droit du travail, à l'époque de la première révolution industrielle, est historiquement lié à l'usine comme pierre angulaire. Naturellement, l'apparition de l'électricité et l'automatisation de la production ont dessiné les contours du monde du travail tel que nous le connaissons aujourd'hui. Toutefois, la révolution 4.0 pourrait entraîner des changements d'une grande ampleur, susceptibles de faire vaciller les fondements mêmes de notre conception du travail, des travailleurs et de la législation du travail.

La numérisation et l'interconnexion des processus, assorti aux possibilités infinies d'utilisation des systèmes de communication et de traitement des données, ont à jamais effacé les frontières nationales de la production. C'est pourquoi, nous proposons une réflexion sur ce nouveau concept de "cybermigrants ${ }^{1}$, nés de la révolution industrielle 4.0, qui font le choix d'une prestation de services en temps réel et par-delà les frontières.

Depuis des décennies, l'activité de production nationale est transférée vers des pays où le coût de production est(largement) inférieur, ceci afin de maximiser la rentabilité du capital. Force est de constater que les coûts liés à la main-d'œuvre sont d'une importance cruciale. C'est pourquoi, la plupart des entreprises cherchent des sites où la main-d'œuvre est moins chère que celle du pays d'origine, pour y délocaliser la production de leurs biens, que ce soit en partie ou en totalité.

La révolution industrielle 4.0 donne une autre dimension à cette approche. La nouveauté actuelle réside dans le fait que ce processus, autrefois lié à la production de biens matériels, s'applique désormais aux services.

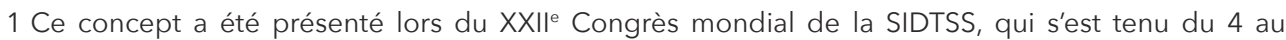
7 septembre 2018 à Turin (Italie) à l'occasion de la présentation de l'ouvrage de A. de la Riva, Cybermigrants, franchir les frontières de la réalité virtuelle: http://www.islssltorino2018.org/ congress/. Cette étude tente d'approfondir la réflexion initiée par S. Strange, "Presidential Address ISA as a Microcosm », International Studies Quarterly, 1995, vol.39, n³, p. 289: "To make any kind of marks, I have to develop ideas for my own, and take a chance that no one would listen (...) Have the courage to do your own thing, and say what you really think. Why else should anyone want to be an academic?». 
II n'y a pas si longtemps encore, la prestation de services avait lieu nécessairement à l'endroit même où ce service était fourni, et généralement de façon quasi instantanée. Les temps ont changé. Aujourd'hui, la prestation de services en temps réel peut être fournie à distance. Par exemple, si j'égare ma carte de crédit et qu'il me faut signaler sa perte, je contacte un numéro de téléphone, mon interlocuteur vit probablement dans un pays autre que le mien et sa langue maternelle est certainement différente de la mienne. Je bénéficie néanmoins du service instantanément, en dépit du fait que le travailleur se trouve à des milliers de kilomètres, voire sur un autre continent. Le service est fourni en temps réel et " réellement » à l'intérieur des frontières de mon pays².

La délocalisation de la prestation de services, rendue possible par les nouvelles technologies ${ }^{3}$, caractérise en grande partie la révolution industrielle 4.0. C'est la raison pour laquelle cette façon de travailler à distance, tout en proposant un service dans des conditions similaires et en produisant la même valeur que s'il était fourni in situ, mérite un concept adapté: le cybermigrant, ici exploré à partir de la cybermigration (I) qui affecte un être humain travaillant par écran (II) et qui, pour cette raison, doit pouvoir être appréhendé sur un plan juridique (III).

\section{I - LA CYBERMIGRATION}

Ce nouveau concept s'inscrit au croisement de trois notions: le télétravail, le « eTravail » et le travail migrant.

La notion de télétravail ${ }^{4}$ renvoie généralement à deux éléments essentiels: d'une part, un travailleur qui se trouve en dehors du siège de l'entreprise et d'autre part, l'utilisation intensive des technologies de la communication et de l'information comme moyens essentiels pour mener à bien la tâche à accomplir.

Le «eTravail» se présente comme une forme plus poussée de télétravail, du point de vue subjectif - car il peut être exécuté selon des modalités contractuelles qui ne s'insèrent pas nécessairement dans le cadre d'un travail protégé par le droit du travail- comme d'un point de vue objectif, parce qu'il n'a pas vocation à être effectué dans les bureaux du donneur

2 Voir notes de bas de page 8 et 9.

3 Selon le rapport de I'OIT, Un monde des affaires en évolution: nouvelles opportunités pour les organisations d'employeurs et d'entreprises, Bureau des activités pour les employeurs (ACT/EMP), 26/03/2019, «56\% des personnes interrogées considèrent l'innovation technologique comme la tendance mondiale ayant l'impact global le plus important sur les entreprises ». Aujourd'hui, l'innovation technologique ouvre l'accès à de nouveaux marchés, crée des opportunités pour de nouveaux modèles d'affaires numériques, accélère le développement de nouveaux produits et services, permet le déploiement de nouvelles méthodes de travail (comme les équipes virtuelles internationales et le télétravail) et étend les réseaux numériques des fournisseurs et des partenaires commerciaux.

4 Idée développée à partir du concept de travail à domicile. A titre d'exemple, citons la Convention internationale du travail $n^{\circ} 177$ de 1996 (en vigueur depuis le 22 avril 2000) et la Recommandation n 184 de 1996, d'où il ressort que le télétravail se retrouverait piégé si l'on inclut la fourniture de services comme contenu possible du travail à domicile. Voir R. Gallardo Molla, Le travail à domicile ancien et nouveau: de la machine à coudre à l'ordinateur, Móstoles, Madrid, 1998, qui insiste sur la nécessité d'une protection globale du travail à domicile, fondée sur la protection d'éléments fondamentaux comprenant à la fois le travail à distance ancien et moderne. 
d'ordre, ni au domicile du prestataire de services, ni dans tout autre espace physique. En résumé, cette forme de travail comprendrait tous les types de tâches réalisées par l'usage intensif de moyens technologiques ${ }^{5}$.

Comme évoqué précédemment, les deux notions de télétravail et de "eTravail» convergent avec une troisième, celle du travailleur migrant. Le travail a toujours été lié aux migrations. Ces dernières ont bien sûr été façonnées tout au long de l'histoire de l'Humanité en quête de nouveaux horizons ou pour diverses raisons sociologiques, politiques, économiques, ethniques ou de conflits. Ces mouvements migratoires tangibles et visibles n'ont jamais cessé depuis l'époque biblique; au contraire, ils se sont développés et constituent un trait caractéristique de notre époque. Ils compliquent le paysage économique, social et culturel des pays d'accueil et des pays de départ, éveillant probablement le meilleur comme le pire dans notre façon de penser et d'agir en tant qu'êtres humains.

Ce nouveau concept, situé au croisement des trois notions évoquées précédemment, implique de concevoir le cybermigrant comme une personne physique qui, sans ancrage dans l'entreprise, est établie hors des frontières nationales et fournit, grâce à un support technologique indispensable et essentiel, un service pour le compte d'une autre personne physique qui la dirige, la contrôle et la rémunère.

Comme chacun le sait, le concept de cybermigrant est par essence indissociable de la délocalisation. En effet, il désigne une personne physique vivant par-delà les frontières du pays où le service est effectivement fourni, et travaillant en dehors de l'entreprise qui la rémunère ${ }^{6}$. Autrement dit, le cybermigrant ne s'inscrit physiquement ni dans un espace commercial, ni dans le pays où le service est fourni à son destinataire. La séparation physique est donc totale entre l'employeur et le travailleur, de même qu'entre le bénéficiaire du service et le travailleur qui exécute la prestation. Mais cette double séparation physique est compensée par une hyperconnexion virtuelle et double elle aussi. C'est pourquoi, comme indiqué précédemment, le soutien technologique est indispensable et essentiel.

L'élément technologique est celui qui permet d'exécuter la tâche assignée ${ }^{7}$ dans la mesure où, à défaut, le travail ne peut être accompli dans le cadre de la cybermigration ${ }^{8}$ même s'il peut évidemment l'être sous la forme traditionnelle du travail en entreprise, comme

5 W. Sanguinetti Raymond, «Télétravail »: https://wilfredosanguineti.files. wordpress.com/2008/11/ teletrabajo-voz-dic-montoya-wsanguineti.pdf

6 II faut préciser que le cybermigrant ne propose pas ses services à un call center, ni à une succursale de l'entreprise qui l'embauche, car sinon il s'agirait d'un travailleur typique dans une entreprise classique, assujetti à la réglementation locale.

7 II est essentiel, primordial. A défaut, il n'y a aucun moyen d'accomplir la tâche demandée, à moins d'être physiquement dans l'entreprise.

8 Imaginez un hôpital qui effectue des études médicales dont l'aspect clinique est pris en charge dans l'hôpital lui-même, tandis que le rapport est rédigé à distance mais en temps réel. Imaginez un organisme financier qui reçoit dans son bureau une demande de crédit dont l'analyse sera effectuée par un agent qui se trouve à des milliers de kilomètres. Imaginez une société de production cinématographique qui engage un professionnel du son, installé de l'autre côté de l'océan, pour faire la bande sonore de tous ses films. Imaginez un constructeur automobile asiatique qui confie à un expert latino-américain la traduction de tous les livrets d'instructions des véhicules. 
c'était le cas jusqu'à présent ${ }^{9}$. Ainsi, cette technologie libère le travailleur de son obligation d'ancrage physique au sein d'une entreprise donnée, dans un pays donné, en vue de fournir localement un service à un bénéficiaire. Dès lors, les cybermigrants travaillent directement pour l'entreprise, en dehors du siège de celle-ci et parfois même depuis un autre continent. Ils effectuent leurs tâches dans le cadre d'échanges interactifs (two way line), conformément aux nouvelles formes d'organisation du travail transnational globalisé. II ne s'agit donc pas d'un travail différent, mais d'une manière différente d'effectuer la prestation de service, caractérisée aujourd'hui par l'absence de communauté physique.

Comme évoqué ci-avant, c'est précisément pour cette raison que la séparation physique trouve son pendant dans l'hyperconnexion virtuelle, présente elle aussi à deux niveaux. Tout d'abord, au niveau de l'employeur, dans la mesure où les éléments nécessaires à la protection du travailleur par le droit du travail sont présents, à savoir le pouvoir de direction, de contrôle, de rémunération, et le travail pour le compte d'autrui. Ces éléments sont évidemment à l'image de la relation particulière à l'employeur et s'adaptent aux spécificités liées à l'exécution des tâches et aux modalités particulières d'exercice de l'autorité. C'est pourquoi certains concepts, tels que celui de subordination, exigent une approche plus ouverte ${ }^{10}$. Néanmoins, cela ne signifie pas qu'on ne retrouve pas chez les cybermigrants certains aspects des concepts traditionnels, à l'instar de la subordination classique du travailleur associée à la première révolution industrielle. Les cybermigrants sont souvent soumis à un contrôle encore plus strict de la part de l'employeur que la plupart des travailleurs de l'époque, un contrôle favorisé, en outre, par la technologie.

Par ailleurs, I'hyperconnexion virtuelle est aussi présente au niveau de la relation avec le consommateur. Le travailleur et le consommateur sont liés par ce service fourni, non pas en «face à face » mais à travers des octets. Une hyperconnexion qui se caractérise par une possibilité d'interaction illimitée, sans barrières de temps, d'espace, d'horaires ou de lieu.

La situation pourrait donc se résumer en quelques mots: délocalisation et séparation physique, hyperconnexion virtuelle, technologie informatique au service du travail à accomplir.

\section{II - LA PRÉSENCE D'UN ÊTRE HUMAIN DERRIÈRE L'ÉCRAN}

Ces travailleurs étrangers établis par-delà les frontières accomplissent la même tâche que s'ils étaient physiquement présents au moment de l'exécution de la prestation de service, comme c'était le cas traditionnellement.

Cette situation est rendue possible grâce à la technologie qui élimine les frontières physiques et nous place dans un espace unique, à la fois global et virtuel, au sein duquel le prestataire de services reste invisible, anonyme derrière son écran. Or, il convient de souligner qu'un prestataire installé dans un pays dont le talent est immédiatement exploité dans un

9 Toutes les tâches mentionnées dans la note précédente étaient à l'époque, et encore certainement dans bien des cas aujourd'hui, accomplies au sein de l'entreprise elle-même. Le médecin chargé du rapport n'était pas seulement à l'hôpital, il était peut-être même aussi dans la salle où les images à étudier avaient été prises, l'analyste de crédit avait sûrement son bureau à quelques pas de là où la demande de prêt était envoyée, le technicien du son travaillait sans doute dans les studios de cinéma et le traducteur au sein des bureaux administratifs de l'usine automobile. Avec le recours à la technologie, bien sûr, ces tâches pouvaient aussi être réalisées sur place par des travailleurs -souvent étrangers. Dans ce cas, il ne s'agit pas de cybermigrants, mais de travailleurs fournissant un service localement, qu'ils aient ou non la nationalité du pays en question, qu'il s'agisse de travailleurs migrants types ou non.

10 Pas nécessairement plus floue ni plus complaisante. 
autre pays crée de la richesse dans le pays dans lequel le service est fourni et non pas là où il est produit. Nous sommes donc confrontés à une sorte de migration non pas corporelle ou physique, mais bien de compétences: une «cybermigration ».

Ce cybermigrant, par la valeur ajoutée qu'il apporte au produit de son travail et par son lien de subordination à l'employeur, est identique au migrant qui se déplace physiquement. Finalement, l'employeur retire les mêmes bénéfices de son travail, mais sans avoir les mêmes responsabilités.

Ainsi, le cybermigrant devra être disponible uniquement dans la mesure imposée par l'employeur. Personne ne contrôlera la journée de travail quotidienne ou hebdomadaire, les temps de repos ou le paiement du salaire fixé. Personne ne contrôlera non plus le respect des dispositions légales, puisque le travailleur est invisible à la fois pour l'État dans lequel il réside et pour l'État au sein duquel le service est fourni. Certes, il est vrai qu'en fonction du type de prestations de services, du nombre de prestataires individuels disponibles et des caractéristiques du pays de résidence, les niveaux de salaire et les avantages proposés par les employeurs du pays d'origine varient. La situation n'est évidemment pas la même entre un travailleur qualifié, qui fournit un service hautement spécialisé, et un travailleur classique. Le travailleur qualifié percevra peut-être même un salaire alléchant comparé à celui de ses compatriotes, alors que ceux-ci accomplissent les mêmes tâches localement. Mais une chose est sûre, ce travailleur ne bénéficiera d'aucun statut officiel, pas plus que d'une couverture sociale. De plus, il travaillera probablement en décalé et sans limite horaire.

Sila réalité des droits individuels est difficile à mesurer, celle des droits collectifs l'estencore davantage dans la mesure où les cybermigrants appartiennent à une collectivité totalement floue, pour ne pas dire réduite à une nébuleuse d'octets ${ }^{11}$. Quelle conscience collective peutil y avoir lorsque la prestation de services s'effectue depuis son propre domicile, éloignée de plusieurs milliers voire centaines de milliers d'autres personnes affectées aux mêmes tâches depuis leur domicile mais parfois dispersées aux quatre coins du globe?

Si rien n'incite ces travailleurs à rejoindre leur secteur formel, à quel type de couverture sociale peuvent-ils prétendre? Alors que, partout dans le monde, la protection sociale s'organise pour l'essentiel autour du travail formel, ces travailleurs sont totalement lésés. Ils pourront tout au plus aspirer à des prestations non contributives, généralement peu nombreuses et dont le niveau de couverture reste faible, en particulier dans les pays les moins avancés.

Par leur statut de cybermigrant, ces travailleurs et leurs familles ne pourront bénéficier que d'un accès limité aux services de santé, à l'éducation et aux droits les plus fondamentaux auxquels ils ne peuvent même pas prétendre dans leur propre pays. II est clair qu'aucun avenir ne s'ouvre à eux et à leurs familles, dès lors qu'ils ne peuvent profiter des avantages offerts aux citoyens des pays riches dont ils contribuent pourtant au développement.

Comment répondre alors au besoin de protection juridique du travailleur 4.0 ?

11 Si le problème de la définition du champ d'application subjectif des conventions collectives est déjà perceptible dans le cas des travailleurs classiques qui franchissent momentanément les frontières, imaginez la complexité supplémentaire dans le cas des cybermigrants. Voir A.M. Molina Martín, « Mobilité géographique internationale des travailleurs et champ d'application subjectif des conventions collectives », XXVIIe Journées universitaires d'Andalousie sur le droit du travail et les relations professionnelles, Conseil andalous des relations de travail, 2009, p. 111, qui analyse cette hypothèse du point de vue de la cybermigration, de façon à appréhender le travail des cybermigrants parallèlement à celui des expatriés temporaires, mais dans un cadre temporel illimité. 


\section{III - POUR UNE APPROCHE JURIDIQUE DE LA CYBERMIGRATION}

Comme nous l'avons déjà souligné, la seule différence entre un travailleur qui fournit des services directement au client de son employeur et un travailleur étranger qui fait de même depuis son pays, tient au lieu de résidence. Cette possibilité d'action et de mobilisation par-delà les frontières nationales est due aux nouvelles technologies issues de la révolution industrielle 4.0, des technologies qui, aujourd'hui, règnent en maîtres dans le secteur des services de l'économie mondiale. Le phénomène ici observé, appelé cybermigration, mérite de faire l'objet d'une réglementation qui tienne compte de ses spécificités.

C'est pourquoi, il pourrait être envisageable d'appliquer aux cybermigrants le vaste cadre réglementaire de la migration et, en résumé, de leur assurer la même protection qu'aux travailleurs locaux, à l'abri de toute discrimination en matière d'emploi fondée sur la nationalité. Cette approche permettrait d'aller vers une mondialisation ${ }^{12}$ des services qui soit socialement durable ${ }^{13}$, tout en freinant le dumping social qui frappe à la fois les pays bénéficiaires des services et les pays d'origine, mais aussi les personnes physiques et les entreprises, bien que de manière différente et à des degrés divers.

Si la plupart des traités internationaux n'oblige pas les États à réglementer les activités extraterritoriales de leurs entreprises, l'application des règles relatives au travail des migrants à l'égard de ces prestataires qui fournissent des services depuis l'étranger permettrait un contrôle international, dans le respect des frontières géographiques. Tout comme les octets brouillent les lignes de démarcation souveraine entre les pays, l'application des règles sur le travail des migrants atténue les barrières dans le domaine des droits.

Il ne s'agit donc pas de proposer de nouvelles mesures ayant des implications extraterritoriales à l'égard des entreprises nationales, mais de valoriser les services fournis par une main-d'œuvre extérieure au pays. II ne s'agit pas non plus, loin s'en faut, d'établir une législation nationale dont la portée dépasserait les frontières nationales. En revanche, il s'agit bien d'ôter les œillères qui biaisent notre regard sur les concepts traditionnels et nous empêchent de voir que le concept de cybermigrants n'est pas si éloigné de celui des migrants traditionnels ${ }^{14}$. Une approche plus ouverte sur les réglementations internationales permettrait de les appliquer de façon générale au travail des migrants, peu importe au fond que la migration soit physique ou cybernétique.

12 D'après le rapport du 26/03/2019 Un monde des affaires en évolution: nouvelles opportunités pour les organisations d'employeurs et d'entreprises, Bureau des activités pour les employeurs (ACT/ EMP) de I'OIT: « Malgré les déconvenues récentes, la globalisation de l'économie mondiale est un fait, et elle évolue à un rythme effréné. Les économies émergentes, qui se trouvaient autrefois à la périphérie des chaînes d'approvisionnement mondiales (principalement en tant que fournisseurs de biens à bas coût, de ressources naturelles à l'état brut ou de services de base), deviennent des centres de capitaux, d'innovation et de talent ».

13 Sans cantonner le sujet aux déclarations de principes fondamentaux, aux actions de responsabilité sociale des entreprises, ou aux avancées de ce que l'on appelle la soft law, sans toutefois minimiser leur pertinence dans ce domaine où elles ont joué un rôle de premier plan dans l'endiguement de l'activité professionnelle et la jouissance de certains droits fondamentaux. Ces sujets ont été analysés en profondeur par I. Daugareilh, "La responsabilité sociale des entreprises transnationales: analyse critique et prospective juridique », Cuadernos de Relaciones Laborales, 2009, 27, n 1 , p. 77.

14 La recommandation de "prendre des mesures novatrices pour faire face à la diversité croissante des formes de travail et notamment au phénomène émergent du travail numérique » est donc particulièrement appropriée, de sorte que les concepts développés ici, bien qu'ils aient été conçus avant le rapport, lui font, humblement, honneur. Travailler pour un avenir meilleur, Commission mondiale sur l'avenir du travail Bureau international du travail, Genève, BIT, 2019, p. 15. 
L'ensemble du système universel des droits de l'homme ${ }^{15}$ offre des solutions acceptables, parfaitement transférables à cette nouvelle modalité de prestation de services. En ce sens, et par-delà ses faiblesses, le système de régulation internationale mis en place par l'Organisation Internationale du Travail, comme cadre éthique commun, est tout à fait pertinent pour protéger les droits fondamentaux à l'ère où la mondialisation affecte désormais tous les aspects du travail humain ${ }^{16}$.

\section{Conclusion}

La nouvelle catégorie de travailleurs migrants, née de l'avènement de la révolution industrielle 4.0 qui a rendu possible la prestation de services par-delà les frontières, pose de sérieux défis et exige de nouvelles approches. Cette façon inédite, et néanmoins courante, d'intervenir dans le secteur tertiaire de l'économie mondiale a touché - à plus ou moins grande échelle - les États, les travailleurs, les entreprises et les syndicats. Elle a également eu un impact sur divers domaines, comme les droits fondamentaux, la couverture sociale, l'écart entre les sexes et le fossé intergénérationnel.

La cybermigration dont il est ici question, ainsi que la nature juridique du concept de cybermigration et la possibilité de la réglementer par divers systèmes juridiques internationaux conçus traditionnellement pour le travail des migrants, se veulent une réponse appropriée aux défis que pose cette nouvelle situation, notamment dans le cadre du « mandat d'universalité » de l'Organisation Internationale du Travail.

Il n'y a pas de meilleur moment pour agir que le moment présent. II n'y a pas de meilleur moment que le présent, si nous faisons les choses correctement. II suffit d'avoir le courage de porter un regard innovant et de puiser au sein des normes traditionnelles de nouvelles manières d'agir.

15 Outre les principaux pactes et traités universels et régionaux relatifs aux droits de l'homme, deux documents mériteraient selon l'auteur, d'être mieux connus: "Principes directeurs sur les entreprises et les droits de l'homme», ONU, 2011; "Principes directeurs de l'OCDE pour les entreprises multinationales ", OCDE, 2013: http://dx.doi.org/10.1787/9789264202436-es" révision 2011

16 J-M. Servais, «Labour Law. Its role, trends and potential », Labour education, 2006/2-3, n 143.

\section{AMALIA DE LA RIVA}

Docteur en droit et en sciences sociales, Professeur de droit du travail et de la sécurité sociale, Université de la République, Uruguay

Membre du conseil d'administration de l'Association uruguayenne du droit du travail et de la sécurité sociale, Coordinateur de I'Unité Projets, Coopération et Accords de la Faculté de Droit de I'Université de la République, Directeur de l'Institut de droit du sport du Barreau de I'Uruguay.

Thèmes de recherche: droit du travail, droit individuel et collectif, droit du sport.

Publications:

A. de la Riva, «E-sports: mucho más que un juego », Revista de Direito do Trabalho, ed. spéciale Cielo Laboral, 2018, Uruguay.

$\sim$ A. de la Riva, «Tres cuestiones sobre la prescripción de créditos laborales. Interrupción. Inicio del cómputo. Plazo », Revista de doctrina, jurisprudencia e informaciones sociales, tome 61, $n^{\circ} 270,2018$. 